OPEN ACCESS

Edited by: Rebeca Martín,

INRA Centre Jouy-en-Josas, France

Reviewed by:

Maria Aponte,

University of Naples Federico II, Italy

Natalia Martins Breyner,

McMaster University, Canada

*Correspondence:

Tom Van de Wiele

tom.vandewiele@ugent.be

Specialty section:

This article was submitted to

Food Microbiology,

a section of the journal

Frontiers in Microbiology

Received: 30 June 2017 Accepted: 14 September 2017

Published: 29 September 2017

Citation:

El Hage R, Hernandez-Sanabria E and Van de Wiele T (2017) Emerging

Trends in "Smart Probiotics":

Functional Consideration

for the Development of Novel Health

and Industrial Applications.

Front. Microbiol. 8:1889.

doi: 10.3389/fmicb.2017.01889

\section{Emerging Trends in "Smart Probiotics": Functional Consideration for the Development of Novel Health and Industrial Applications}

\author{
Racha El Hage, Emma Hernandez-Sanabria and Tom Van de Wiele* \\ Center for Microbial Ecology and Technology, Faculty of Bioscience Engineering, Ghent University, Ghent, Belgium
}

The link between gut microbiota and human health is well-recognized and described. This ultimate impact on the host has contributed to explain the mutual dependence between humans and their gut bacteria. Gut microbiota can be manipulated through passive or active strategies. The former includes diet, lifestyle, and environment, while the latter comprise antibiotics, pre- and probiotics. Historically, conventional probiotic strategies included a phylogenetically limited diversity of bacteria and some yeast strains. However, biotherapeutic strategies evolved in the last years with the advent of fecal microbiota transplant (FMT), successfully applied for treating CDI, $\mathrm{IBD}$, and other diseases. Despite the positive outcomes, long-term effects resulting from the uncharacterized nature of FMT are not sufficiently studied. Thus, developing strategies to simulate the FMT, using characterized gut colonizers with identified phylogenetic diversity, may be a promising alternative. As the definition of probiotics states that the microorganism should have beneficial effects on the host, several bacterial species with proven efficacy have been considered next generation probiotics. Non-conventional candidate strains include Akkermansia muciniphila, Faecalibacterium prausnitzii, Bacteroides fragilis, and members of the Clostridia clusters IV, XIVa, and $\mathrm{XVIII.} \mathrm{However,} \mathrm{viable} \mathrm{intestinal} \mathrm{delivery} \mathrm{is} \mathrm{one} \mathrm{of} \mathrm{the} \mathrm{current} \mathrm{challenges,} \mathrm{due} \mathrm{to} \mathrm{their}$ stringent survival conditions. In this review, we will cover current perspectives on the development and assessment of next generation probiotics and the approaches that industry and stakeholders must consider for a successful outcome.

Keywords: FMT, next generation probiotics, bacterial consortium, synthetic community, CDI

\section{INTRODUCTION}

The gut microbiota plays a significant role in human health, participating in several functions beneficial to the host (Patel and DuPont, 2015; Kristensen et al., 2016). It has been implicated in preventing pathogen colonization (Hand, 2016), shaping our immune system (Round and Mazmanian, 2009; Patel and DuPont, 2015; Macpherson et al., 2017), stimulating the production of gastrointestinal hormones (Saulnier et al., 2013), and regulating brain behavior (De Palma et al., 2014, 2017) through production of neuroactive substances (Steenbergen et al., 2015; Kristensen et al., 2016). 
Additionally, the gut microbiota has been involved in the fermentation of non-digestible carbohydrates reaching the colon. This process leads to the production of short chain fatty acids (SCFAs), which elicit health benefits (den Besten et al., 2013). The human gut microbiota can be manipulated through either passive or active processes. Passive factors include hygiene, lifestyle, and diet. For instance, primary colonizers of the gut involved in the immune development are shifted by sanitary practices (Zhou, 2016). In addition, dietary constituents can promote phylogenetic variations in the microbiota (Graf et al., 2015). In this context, prebiotics are defined as "a substrate that is selectively utilized by host microorganisms conferring a health benefit" (Gibson et al., 2017). Prebiotics act as growth substrates (Patrascu et al., 2017) to enhance the activity of bacterial genera (Scott et al., 2015) such as bifidobacteria and butyrate-producing clostridia (Rivière et al., 2016). SCFA and vitamins resulting from the fermentation of these components are crucial for human health (Graf et al., 2015). In terms of lifestyle factors, physical activity is known to positively impact the diversity of gut microbiota. In fact, gut microbiota of athletes is more diverse than that of non-athletic subjects (Clarke et al., 2014). Amongst the active processes manipulating microbiota composition are antibiotics and probiotics. Antibiotic use has been linked to dysbiosis (Langdon et al., 2016), even leading to low diversity, evenness, and taxonomic richness (Dethlefsen and Relman, 2010; Francino, 2016). Moreover, presence and expression of microbial genes are altered following antibiotic therapy (Reijnders et al., 2016). These detrimental outcomes may lead to decreased SCFA, glycolysis, vitamin production, homeostasis of the immune system, and impaired protection against pathogens (Guarner and Malagelada, 2003). As a result, antibiotic associated diarrhea (AAD) and recurrent infectious diseases like Clostridium difficile infection (CDI) may occur (Francino, 2016).

On the other side of the spectrum are probiotics, which can affect the host either directly or through their products, or even influence the activity of resident bacteria in the host (Scott et al., 2015). Probiotics are defined as "live microorganisms which when administered in adequate amounts confer a health benefit on the host" (WHO/FAO, 2006; Hill et al., 2014). The effect of probiotics in preventing metabolic syndromes such as obesity, type 2 diabetes (Kasińska and Drzewoski, 2015), and dyslipidemia has been reported (Asemi et al., 2013). For instance, administration of Bifidobacterium (Yin, 2010; Chen et al., 2011; Plaza-Diaz et al., 2014; Reichold et al., 2014; Savcheniuk et al., 2014; Wang et al., 2014) and Lactobacillus species reduced body weight gain and adipose tissue in mice fed high-fat diet through stimulation of adiponectin production (Kim et al., 2013; Kobyliak et al., 2016). In addition, lactobacilli have been proven to have therapeutic effects in different pathologies (Di Cerbo et al., 2016). Moreover, probiotics regulate the mucosal immune response (Klaenhammer et al., 2012), improving the activity of macrophages (Sang, 2010) and changing the expression of the genes associated. Even though these outcomes depend on specific bacteria and strains, probiotics may interact with TLR and downregulate the expression of NF- $\mathrm{NB}$ and pro-inflammatory cytokines
(Ng et al., 2009; Plaza-Diaz, 2014). For instance, peptides of microbial anti-inflammatory molecules (MAMs) that are found in the Faecalibacterium prausnitzii supernatant inhibit the NF-кB pathway in vitro and in vivo (Breyner et al., 2017), confirming the anti-inflammatory and therapeutic properties of F. prausnitzii (Martín et al., 2014). These properties and protective effects of $F$. prausnitzii were identified in different models such as dinitrobenzene sulfate (DNBS)-induced colitis model, dextran sodium sulfate (DSS)-induced colitis (Breyner et al., 2017), and 2,4,6-trinitrobenzenesulfonic acid (TNBS)induced acute colitis in mice (Miquel et al., 2015). Additionally, levels of anti-inflammatory cytokines and immunoglobulins, immune cell proliferation, and production of proinflammatory cytokines produced by the $\mathrm{T}$ cells may be modulated following probiotic supplementation (Miettinen et al., 1996; Nazemian et al., 2016). Furthermore, probiotics can be alternative strategies for inflammatory disorders, as they upregulate the production of CD4+Foxp3+ regulatory $\mathrm{T}$ cells ( $\mathrm{T}_{\text {regs }}$ ) (Kwon et al., 2010; Yan and Polk, 2011).

Different effects on the immune function may be speciesand strain-related (Klaenhammer et al., 2012). It has been reported that probiotics have therapeutic effect on the central nervous system by reducing the intestinal inflammation. In this way, the regulation of HPA axis and the activity of the neurotransmitters may be improved (Wallace and Milev, 2017). Probiotics from Bifidobacterium and Lactobacillus genera are usually delivered through fermented products such as yogurts, milk, and cheeses, or they can be delivered as food supplements (Besseling-van der Vaart et al., 2016).

\section{MONOSTRAIN AND MULTISTRAIN PROBIOTICS}

Probiotics have been categorized into monostrain or multistrain/multispecies products (Timmerman et al., 2004). Different studies have confirmed positive effects on health when multistrain probiotics are used, due to the symbiosis among strains (Timmerman et al., 2004). Strains in multispecies probiotics can be from different genera. For instance, the efficacy of the multispecies probiotic consortium VSL\#3 (Streptococcus thermophilus, Eubacterium faecium, Bifidobacterium breve, Bifidobacterium infantis, Bifidobacterium longum, Lactobacillus acidophilus, Lactobacillus plantarum, Lactobacillus casei, and Lactobacillus delbrueckii subspecies bulgaricus) was proven for the treatment of ulcerative colitis (Venturi et al., 1999; Timmerman et al., 2004). Besides, VSL\#3 supplementation in women with gestational diabetes mellitus (GDM) may help regulate inflammatory markers and positively influence glycemic control (Jafarnejad et al., 2016). In addition, Chapman et al. (2011) described that probiotic mixtures were more effective than single-strain probiotics in inhibiting pathogen growth and atopic dermatitis, suggesting further application on other diseases like IBD. Another multispecies probiotic called Ecologic ${ }^{\circledR}$ Tolerance/Syngut ${ }^{\mathrm{TM}}$ was developed using four different probiotic strains (Bifidobacterium lactis W51, 
L. acidophilus W22, L. plantarum W21, and Lactococcus lactis W19). Strains of this consortium have been proven to strengthen the gut barrier function, have beneficial effects on post-immunological induced stress, inhibit Th2, and stimulate IL-10 levels, thus providing beneficial effects in patients with food intolerance (Besseling-van der Vaart et al., 2016). Moreover, a multispecies probiotic consortium, Ecologic AAD (B. bifidum W23, B. lactis W18, B. longum W51, E. faecium W54, L. acidophilus W37 and W55, L. paracasei W72, L. plantarum W62, L. rhamnosus W71, and L. salivarius W24), reduced diarrhea-like bowel movements when administered in healthy volunteers taking amoxicillin (Koning et al., 2008). Multispecies probiotics also prevented rise in fasting plasma glucose (FPG), to decrease high sensitivity C-reactive protein (hs-CRP), and to increase plasma glutathione (GSH) in diabetic patients (Asemi et al., 2013). van Minnen et al. (2007) provided evidence that manipulation of the intestinal flora with multispecies probiotics reduced bacterial translocation, morbidity and mortality in a rat model of acute pancreatitis. Furthermore, multispecies probiotics rapidly relieved IBS symptoms and shifted the microbiota composition (Yoon et al., 2013). According to these results, combining specific probiotic effects from diverse strains can lead to an additive and more synergetic multispecies probiotic consortium (Timmerman et al., 2007).

However, the phylogenetic origin of probiotics is currently limited to conventional formulations of Bifidobacterium, Lactobacillus species and other lactic acid bacteria (LAB) (Govender et al., 2013) or yeast strains. This may decrease the probiotic effectiveness in the prevention or therapy of diseases entailing severe dysbiosis. Hence, a functionally and phylogenetically diverse probiotic product may be desirable when alterations in the gut microbiota composition are present (Marotz and Zarrinpar, 2016). For instance, CDI and recurrent CDI are major medical conditions that need urgent treatment when conventional antibiotics fail. As a result, development of complex communities with targeted functions is needed.

\section{THE DILEMMA OF FECAL MICROBIOTA TRANSPLANT (FMT)}

Fecal microbiota transplant (FMT) or fecal bacteriotherapy is an alternative strategy successfully used for the treatment of CDI (Kelly, 2013). Severe antibiotic therapy and CDI trigger dysbiosis, reducing diversity and functionality of the gut endogenous microbiota (Brandt, 2012). In this case, C. difficile spores can germinate, colonize, and thrive in the gut. Treatment of CDI requires additional antibiotics, increasing the risk of recurrent CDI (rCDI) after cessation of treatment, as a result of the dysbiosis caused by antibiotic therapy (Becattini et al., 2016; Francino, 2016), due to infection with the original strain (Barbut et al., 2000; Marsh et al., 2012) or re-infection caused by a different strain (Johnson et al., 1989; Kelly, 2009; Figueroa et al., 2012).
Poor colonization resistance from the gut microbiota and the patient's poor immune response further contribute to CDI risk (Pérez-Cobas et al., 2015). Recurrent CDI risk is 10-20\% after initial CDI (Surawicz et al., 2013), and it increases to $45 \%$ after a first relapse, and to $60 \%$ for those with two or more recurrences (Bartlett, 1990). However, FMT can resolve both CDI and rCDI (Bakken, 2009), with a success rate of $90 \%$ when further antibiotic treatments fail (Youngster et al., 2014; Rao and Safdar, 2015). Given the success of FMT, it is now being considered as potential treatment for disorders such as ulcerative colitis (Shi et al., 2016), irritable bowel syndrome (Distrutti et al., 2016), and metabolic syndrome (Hartstra et al., 2015). For instance, FMT induced remission in patients with active ulcerative colitis (Moayyedi et al., 2015), potentially as a result of the introduction of normal flora and the subsequent correction of the imbalance in the microbiota caused by the disease (Bakken et al., 2011). The complexity of the fecal sample can be the key factor behind the positive shift in the microbiota composition generated by the FMT (Marotz and Zarrinpar, 2016). Thus, diversity of the donor microbiome may be crucial (Leszczyszyn et al., 2016). Indeed, some patients do not respond to FMT, probably because only specific bacterial phylotypes can be therapeutic when effectively transferred (Vermeire et al., 2015). Hence, FMT efficacy for treating gastrointestinal disorders is controversial (Sbahi and Di Palma, 2016). Adverse effects after FMT include nausea, vomit, fever, abdominal pain, and diarrhea (Vermeire et al., 2015; Pigneur and Sokol, 2016). Data for long-term effects of FMT is lacking, but theoretically, any disease phenotype from the donor can be transferred to the patient (Sbahi and Di Palma, 2016). This could be expected, as the uncharacterized nature of FMT may result in undetected or unmonitored risk factors such as viruses, pathogens or even allergens being passed to the FMT recipient, causing disease. To overcome this problem, Petrof et al. (2013) developed a synthetic bacteria cocktail with characterized nature to substitute FMT. Alternatively, a thorough pre-screening should be performed on the donor before the actual procedure of the FMT. Thus, the French Group of Fecal microbiota Transplantation (FGFT) was created to secure and evaluate the practice in this field (Sokol et al., 2016). Despite having experience treating CDI, FMT is not yet the top treatment choice of physicians (Zipursky et al., 2014). However, the majority of gastroenterologists and physicians in metropolitan areas were supportive to the idea of creating a fecal transplantation center, and a high percentage of the physicians would refer their patients to those centers (Jiang et al., 2013).

\section{ALTERNATIVES FOR FECAL MICROBIOTA TRANSPLANT (FMT)}

Additional microbiome therapeutics using characterized microbial communities of selected fecal bacteria could be developed to replace FMT, and yield the desired outcome (Sbahi and Di Palma, 2016). For instance, Petrof et al. (2013) described a stool substitute constituted by 33 different purified 
TABLE 1 | Strains composing the RePOOPulate consortium.

Composition of Stool Substitute (RePOOPulate)

Acidaminococcus intestinalis

Bacteroides ovatus

Bifidobacterium adolescentis (two different strains)

Bifidobacterium longum (two different strains)

Blautia producta

Clostridium cocleatum

Collinsella aerofaciens

Dorea longicatena (two different strains)

Escherichia coli

Eubacterium desmolans

Eubacterium eligens

Eubacterium limosum

Eubacterium rectale (four different strains)

Eubacterium ventriosum

Faecalibacterium prausnitzii

Lachnospira pectinoshiza

Lactobacillus casei/paracasei

Lactobacillus casei

Parabacteroides distasonis

Raoultella sp.

Roseburia faecalis

Roseburia intestinalis

Ruminococcus torques (two different strains)

Ruminococcus obeum (two different strains)

Streptococcus mitis

intestinal bacteria isolated from a healthy donor (Table 1), to treat rCDI. In this study, the synthetic bacterial mixture was infused through the colon of the infected patient causing a change in the stool microbial profile. Major shifts reflecting the isolates of the synthetic mixture were still detectable 6 months after treatment. Thus, the concept of "RePOOPulate" the gut microbiome was coined. Authors of the study suggested that using a synthetic stool substitute may be an effective method to replace the use of FMT for treating rCDI. Although further validation is needed, complete resolution of the infection was achieved. Several advantages of this synthetic stool substitute can be highlighted. The composition of the administered bacterial cocktail is accurately characterized, facilitating registration. Further, assembly of the synthetic bacterial cocktail is highly reproducible enabling standardization and upscaling. In addition, patient safety can be guaranteed, because the bacterial mixture can be rendered pathogen- and virus-free (Petrof et al., 2013). These data suggest that a multi-species community such as that in the RePOOPulate study, can be more effective than singlestrain probiotics or mixed cultures of probiotic species. This can be because the RePOOPulate community preserved its structure and thus successfully colonized a new environment (Petrof et al., 2013). Moreover, RePOOPulate consisted of a more phylogenetically diverse community including strains with beneficial health effects that can be candidates for next generation probiotics.

\section{NEXT GENERATION PROBIOTICS}

Looking at its internationally recognized definition, probiotics are live microorganisms that, when administered in adequate numbers, confer health benefits on the host. Probiotics are usually isolated from our commensal gut bacteria, but cannot be given the definition of probiotics until their stability, content, and health effect are characterized (Sanders, 2008). Probiotics are thought to improve the balance in the host, prevent disturbances, and decrease the risk of pathogen colonization (Goldenberg et al., 2013). They have been referred to as functional foods or beneficial bacteria, and they have been considered for the prevention and treatment of $C$. difficileassociated diarrhea (CDAD) (Goldenberg et al., 2013). Probiotics can be found as capsules or food supplements in health food stores and supermarkets (Goldenberg et al., 2013). Pattani et al. (2013) reported that Lactobacillus-based formulations combined with antibiotics reduced the risk of AAD and CDI. They however, suggested that larger studies are needed to decide on the use of probiotic/antibiotic combination as a therapy over the single species probiotic (Pattani et al., 2013). Furthermore, findings from randomized control trials (RCTs) and meta analyses suggest that there is moderate evidence on the ability of probiotics to prevent primary CDI (people at risk of CDI), but there is no enough evidence suggesting the probiotics can prevent secondary CDI (recurrent CDI) (Evans and Johnson, 2015). There are still some evidence gaps for the use of probiotics in the prevention of CDI such as the interaction between specific classes of antibiotics with the probiotics used on CDI risk, the bacterial taxa that provides the best efficacy in the prevention of CDI, and the use of probiotics in immunocompromised or critically ill patients (Rao and Young, 2017). Hence, future RCT should consider these different concerns (Rao and Young, 2017). Besides, probiotics impact the gut-brain axis.

For example, Bifidobacterium longum NC3001 had beneficial effects on psychiatric comorbidities, which in turn could temporarily improve the quality of life in IBS patients, indicating that this probiotic reduces limbic reactivity (Pinto-Sanchez et al., 2017).

Overall, classical probiotics show limited effects on the human gut microbiota seeking the need for a better selection and formulation of bacterial strains (Neef and Sanz, 2013). Results from previous studies show promising outcomes in the treatment or prevention of diverse metabolic and inflammatory diseases by specific bacteria (Neef and Sanz, 2013). Those probiotics encompass species different from Lactobacillus and Bifidobacterium (Cani and Van Hul, 2015; Patel and DuPont, 2015). Nevertheless, the gut microbiome is a complex community, which makes it difficult to define the host-microbe interaction.

The United Nations Food and Agriculture organization (FAO) definition of probiotics is broad, allowing flexibility in terms of the phylogenetic origin of probiotics. Information generated from previous studies assisted in the selection of next generation probiotics, which include members from Clostridium 
clusters IV, XIVa and XVIII, F. prausnitzii, Akkermansia muciniphila, Bacteroides uniformis (Neef and Sanz, 2013; Patel and DuPont, 2015), Bacteroides fragilis (Round et al., 2011), and Eubacterium hallii (Udayappan et al., 2016). These next generation probiotics were evaluated in preclinical trials and yielded positive outcomes for inflammatory and metabolic disorders (Neef and Sanz, 2013; Patel and DuPont, 2015). In addition, new techniques are required for the development of new probiotic products containing strains from human origin. This is to say, these strains must come from the major groups of the intestinal microbiota, they have to be defined to have a safe status and proven to have potential beneficial effects (Martín et al., 2017). In the following sections, we will discuss some of the most promising bacterial species that are currently under consideration for being used as next-generation probiotics.

\section{Faecalibacterium prausnitzii}

Faecalibacterium prausnitzii is an extreme oxygen sensitive (EOS) bacterium (Martín et al., 2017) belonging to the Clostridium cluster IV, and it accounts for $3-5 \%$ of the total fecal bacteria, and it is one of the predominant groups in the human feces (Breyner et al., 2017). Quévrain et al. (2016) reported low proportions of this species in the fecal and mucosa-associated microbiome in Crohn's disease (CD). F. prausnitzii may possess in vivo and in vitro anti-inflammatory effects. F. prausnitzii may possess in vivo and in vitro anti-inflammatory effects. Breyner et al. (2017) confirmed the anti-inflammatory properties of MAM, and their ability to reduce Th1 and Th17 proinflammatory cytokines in Mesenteric Lymphatic Node (MLN) and colon tissues in both DNBS and DSS colitis model. MAM was also able to improve TGF $\beta$ cytokine which affects $\mathrm{NF}-\kappa \mathrm{B}$ activation in DNBS model thus protecting the host and decreasing intestinal inflammation (Breyner et al., 2017). In addition, F. prausnitzii can induce the Clostridium-specific IL-10-secreting regulatory $\mathrm{T}$ cell subset, present in several human colonic cells. Its capacity for lowering IL-12 and IFN $\gamma$ production indicates that the interaction between $F$. prausnitzii and the host shape and maintain the gut barrier immune function (Quévrain et al., 2016). In this way, anti-inflammatory molecules from $F$. prausnitzii may be used as targeted antiinflammatory drugs for CD. Moreover, MAM could function as a CD biomarker, predicting loss of $F$. prausnitzii functionality. However, further research should be conducted to elucidate the MAM production mechanisms, before considering it for CD management. Sokol colleagues reported that low proportions of F. prausnitzii on a resected ileal Crohn's mucosa were associated with $C D$ recurrence after 6 months. In addition, the oral administration of live F. prausnitzii or its supernatant in mice could reduce the severity of trinitrobenzene sulfonic acid (TNBS) colitis and correct the associated dysbiosis (Sokol et al., 2008). The results from this study suggest that F. prausnitzii can be considered as a promising probiotic candidate for the treatment of pathologies characterized by chronic gut inflammation (Sokol et al., 2008). Besides, all F. prausnitzii strains have proven anti-inflammatory properties, which allows them to further be tested in murine models to determine their beneficial effects before moving to human trials (Martín et al., 2017).

\section{Akkermansia muciniphila}

Recent evidence shows that there is a link between the altered gut microbiota and metabolic diseases like obesity, diabetes mellitus, and cardiovascular disease (Schneeberger et al., 2015; Dao et al., 2016; Li et al., 2016). Higher abundance of A. muciniphila, a mucin degrading microbe, was associated with healthier metabolic status. Everard et al. (2014) and Schneeberger et al. (2015) studied the effects of high fat diet on metabolic parameters and the gut microbiota composition over time, and they found that A. muciniphila was decreased. The negative impact on A. muciniphila was associated with expression of lipid metabolism, inflammatory markers in adipose tissue, and different parameters like increased blood glucose, insulin resistance and plasma triglycerides (Schneeberger et al., 2015). This prompted the research toward investigating the putatively positive role of $A$. muciniphila in adipose tissue homeostasis and metabolism. Dao et al. (2016) assessed clinical parameters and A. muciniphila abundance before and after a 6-week calorie restriction period, followed by stabilization diet. The results of this intervention study indicate that the higher abundance of $A$. muciniphila at baseline was associated with improvement in blood glucose homeostasis, lipid profile, and body fat distribution after the intervention. Thus, A. muciniphila can be used as a prognostic tool for the success of diet interventions (Dao et al., 2016). Moreover, Li et al. (2016) reported that administration of A. muciniphila could reverse the atherosclerotic lesions, improve metabolic endotoxemia-induced inflammation, and ultimately restore the gut barrier.

\section{Bacteroides fragilis and Bacteroides uniformis}

Bacteroides species are commensal bacteria that represent 25\% of our gut bacterial population. They are gram negative, anaerobic, bile resistant, and non-spore forming bacteria. Bacteroides can be passed from the mother to the child during vaginal delivery, thus becoming primary colonizers of the gut. When retained in the gut, Bacteroides act as commensals and can be beneficial for the host (Wexler, 2007). The most common isolate from the clinical specimens is $B$. fragilis, which is the most virulent Bacteroides species (Wexler, 2007).

Bacterial colonization of the gut can greatly affect the immune system, either through the direct host-bacteria interaction, or by molecules produced by our commensal bacteria. B. fragilis produces polysaccharide A (PSA), which is an immunomodulatory molecule that activates the T-cell dependent immune responses (Troy and Kasper, 2010). Those responses are involved in the development and homeostasis of the host immune system (Troy and Kasper, 2010). Furthermore, Round et al. (2011) demonstrated that B. fragilis activates Toll-like receptor (TLR) pathways. This occurs because 
PSA signals through TLR2 on Foxp3+ (forkhead box P3) regulatory $\mathrm{T}$ cells to boost immunologic tolerance. As a result, PSA can be considered as a model symbiosis factor, because it preserves the balance between $\mathrm{T}$ cell types and maintains the immune system homeostasis (Round et al., 2011).

As for Bacteroides uniformis (B. uniformis) CECT 7771, it is considered a potential probiotic strain originally isolated from the feces of healthy breastfed infants. Oral administration of this specific strain in high fat diet-fed mice improved lipid profile, reduced glucose insulin and leptin levels, increased TNF- $\alpha$ production by dendritic cells (DCs) in response to LPS stimulation, and increased phagocytosis (Gauffin Cano et al., 2012). Thus, administration of B. uniformis CECT 7771 can ameliorate metabolic disorder and immunological dysfunction related to intestinal dysbiosis in obese mice (Gauffin Cano et al., 2012; Yang et al., 2016). Furthermore, acute administration of this strain to mice did not promote adverse effects on health status or food intake, and there was no bacteria translocation to blood, liver, or lymph nodes. This indicates that there are no safety concerns for this strain in mice, but further investigation should be completed in humans (Fernández-Murga and Sanz, 2016).

\section{Eubacterium hallii}

Eubacterium hallii is an important anaerobic butyrateproducer resident in our gut, which influences the intestinal metabolic balance (Engels et al., 2016). Butyrate has been proposed to lower mucosal inflammation and oxidative status, strengthen the epithelial barrier function, and modulate intestinal motility in addition to being an energy source for colonocytes (Canani et al., 2011). E. hallii can yield propionate from a broad range of substrates. This versatility may enhance the host-gut microbiota homeostasis (Engels et al., 2016). Moreover, administration of $E$. hallii in obese and diabetic $\mathrm{db} / \mathrm{db}$ mice increased energy metabolism and improved insulin sensitivity. However, increasing dosage of $E$. hallii did not impact body weight or food intake, indicating that this strain can a safe and effective alternative for insulin sensitivity (Udayappan et al., 2016).

\section{COCKTAILS OF Clostridium CLUSTER IV AND XIVA MEMBERS}

As previously described, $\mathrm{T}_{\text {regs }}$ can regulate immune homeostasis and serve as a therapeutic target for different gut inflammatory disorders. Induction of the colonic $\mathrm{T}_{\text {regs }}$ is dependent on special properties of our commensal bacteria. Clostridium spp. belonging to clusters IV and XIVa (also known as Clostridium leptum and coccoides groups, respectively) are exceptional inducers of $\mathrm{T}_{\text {regs }}$ in the colon and can be considered as therapeutic options for IBD and allergies (Atarashi et al., 2011). Previous work indicated that a cocktail of strains isolated for the human gut microbiota can be more effective than a single strain in preventing or treating disease. Thus, Atarashi et al. (2013) isolated 17 strains belonging to Clostridia clusters XIVa, IV, and XVIII from a human fecal sample, which were effective in $\mathrm{T}_{\text {reg }}$ cell differentiation and accumulation in mouse colon. Authors proposed that the SCFAs produced by this community influenced the expression of Foxp3, a key gene controlling $\mathrm{T}_{\text {reg }}$ cell development (Atarashi et al., 2013). Incidentally, Clostridia clusters XIVa and IV are decreased in fecal samples from patients with inflammatory bowel disease (IBD), and thus the cocktail of the 17 strains could potentially reverse this dysbiosis (Atarashi et al., 2013).

\section{INDUSTRIAL APPLICATIONS AND INTERESTS}

\section{Current Developments}

Since the manipulation of the gut microbiota has been proven to be promising to prevent and treat different diseases, pharmaceutical and food industries would be interested in the potential therapeutic approaches described before. For instance, Seres health and Rebiotix companies are working on developing a defined microbial cocktail and a standardized commercially prepared FMT, respectively. These therapeutic approaches are intended to treat CDI, and that can be used as an alternative for FMT. Synthetic microbial communities designed for transplants are expected to meet production, mode of action and safety standards (Orenstein et al., 2015; van der Lelie et al., 2017). For instance, Seres health developed SER109, a novel biological agent proposed to restore the balance in the gut microbiome, promoting resistance to pathogenic invaders like C. difficile (Khanna et al., 2016). Seres health also developed SER-287 for the treatment of IBD and in specific ulcerative colitis (Inflammatory Bowel Disease | Seres Therapeutics, 2017). Rebiotix commercially developed RBX2660, a mix of live human microbes for effective treatment of recurrent CDI (Ramesh et al., 2016). Moreover, other formulations including strains belonging to Clostridia classes IV and XIVa were designed to modulate the immune response (Atarashi et al., 2013). The original community of 17 strains (VE202) was developed by Vendanta Biosciences and Johnson and Johnson, and has provided an effective treatment for autoimmune disorders (Reardon, 2014; Ratner, 2015; van der Lelie et al., 2017).

\section{Technical Challenges}

Several challenges concerning the stability of the probiotic during the probiotic production are still unsolved. Microorganisms require strict conditions to grow, such as specific nutritional media and environmental conditions (suitable temperature, $\mathrm{pH}$, water activity, oxygen content, among others). The product manufacturing and storage processes may impact the viability of the bacterial strains, influencing probiotic stability and properties. In addition, it is fundamental to consider the viability of the probiotics after consumption. Bacterial strains should remain viable at sufficient numbers through the gastrointestinal tract (GIT) passage. Therefore, the selection of optimal culture medium and cell protectants is crucial 
to enhance the efficacy of the probiotic product. Moreover, as most probiotic strains are strict anaerobes or facultative anaerobes, oxygen permeation into carriers should be reduced, or oxygen scavengers should be introduced to reduce the redox potential (Shah et al., 2010). Probiotic bacteria can also be protected by microencapsulation, which has been proposed to improve the stability of the strains and can adapt to the GIT conditions (Heidebach et al., 2012). Nowadays, yogurts and fermented milk are the best-established vehicles for probiotics in the market. However, some probiotic strains are sensitive to the different conditions in fermented products, like oxygen and $\mathrm{pH}$, which can, in turn, affect the stability of probiotics through post-acidification during their storage in the fridge. To minimize this phenomenon, strains that lack the ability to post-acidify should be selected (Damin et al., 2008). As a result, this can cause an economic burden for manufacturers, limiting the addition of probiotics in different products (Gueimonde and Sánchez, 2012). Furthermore, manufacturing the probiotic product in a reproducible manner is a critical aspect (Paulo Sousa e Silva and Freitas, 2014). Several attempts to fix the number of viable probiotic strains throughout the products (Shah et al., 2010) have been attempted, to no avail.

\section{Regulatory Challenges}

Probiotics are classified in different categories across countries. Their names and use as functional foods may vary according to different systems. For instance, probiotics fall in the Qualified Presumption of Safety (QPS) list provided by the European Food Safety Authority (EFSA) and referred to as functional foods since there was no legal definition for probiotics. The market for probiotics as functional foods expanded, as a result of probiotic food products like yogurts and fermented milk (Baldi and Arora, 2015), containing conventional LAB. The QPS list is periodically updated according to the safety assessment of the biological products recommended to be added, and not all can be approved (Scientific Opinion on The Maintenance of The List of QPS Biological Agents Intentionally Added to EFSA Panel on Biological Hazards (BIOHAZ), 2013; Ricci et al., 2017). A similar system applies in the United States as Generally Recognized as Safe (GRAS) products should be approved by the FDA. However, if a probiotic is used as a dietary supplement in the United States, then it is considered as "food" and should be regulated by the Dietary Supplement Health and Education Act (DSHEA). If the probiotic was considered to have therapeutic purpose, the probiotic drug should be proven to be safe and effective to be approved by the FDA. Nevertheless, for both the FDA and EFSA, probiotics cannot be used in health claims. On the other hand, Japan acts as a global market leader, where probiotics are considered as both foods and drugs. According to the Japanese regulations, probiotic products are in different category than foods and Foods for Specific Health Uses (FOSHU). Efficacy claims for probiotic products are prohibited on the labeling until the product gets the permission from the Ministry of Health and Welfare (MHLW) to be considered FOSHU, for which efficacy and safety validation is mandatory. FOSHU categorizes the food claims according to the scientific evidence and the strength of the supporting data provided. The government then divided the FOSHU health claims into subcategories, in which their effect could be in GIT, metabolism, cholesterol moderation, or bone health. Japanese regulations also approve new health claims on a regular basis (Baldi and Arora, 2015).

As the definition and classification of probiotics by regulatory agents throughout the world is different, the status of probiotic products is still uncertain. Thus, reservations about probiotic products claims may arise among regulatory bodies, producers, and consumers. Since the probiotic concept is invading the world, further investigation for probiotic traits is needed. Moreover, most probiotics only include LAB, which possess limited phylogenetic diversity and functionality. Hence, critical update of the screenings required by regulatory agents is urgently needed.

\section{Medical Application}

Despite the different studies and outcomes of FMT, FDA approval in North America has not been granted. At the beginning, FMT was considered as investigational new drugs (INDs), and FDA authorization was mandatory. Currently, patients unresponsive to standard antibiotic CDI therapies can opt for FMT after completing an informed consent, where they are notified that FMT is still under investigation. However, SERES 109 and RBX2660 have been granted the Orphan Drug designation by the FDA (Rebiotix Media, 2015; Seres Therapeutics, 2015). As for the EMA in Europe, the use of FMT for the treatment of CDI has not been yet regulated (van Nood et al., 2014; Lowes, 2016). Yet, FMT is regularly applied to curb infections across Europe, and it is considered in clinical trials for many other pathologies. In the search for safe FMT alternatives, research on microbiotic medicinal products (MMP) is in full development and novel applications are continuously being considered. These MMP developments require novel views and strategies from the scientific world, the industry, the medical field, and the regulatory bodies. In this context, platforms like the Pharmabiotic Research Institute have been created, to facilitate discussion between different stakeholders (Pharmabiotic Research Institute, 2017). Overall, additional research needs to be conducted before using FMT alternatives containing characterized microbial communities and next generation probiotics, to guarantee their safety and reproducible efficacy.

\section{CONCLUSION}

FMT may be replaced with a characterized multispecies bacterial mixture that can be safer, free of allergens or viruses, and capable of treating CDI. With the current in vitro and in vivo data, next generation probiotics hold promise to treat diverse medical conditions, and they can be more effective than single or multi strains of the commercial probiotics. Moreover, several different strains with proven health benefits can also be considered candidates for next generation probiotics and other microbiota-based drugs. However, additional research is required for an increased understanding of the interactions 
among those strains, aiming at producing a successful therapeutic formulation. Research should be conducted to demonstrate whether these probiotics can be applicable to humans, as safety assessments have only been completed in animals. Effective carriage of bacterial strains in food matrices is critical for survival. Thus, optimisation of the growing conditions, and even encapsulation must be considered to promote delivery and release of the live product in the colon. The development of next generation probiotics and MMPs hold promise for innovation in both the food/feed sector and the pharmaceutical industry. A close interaction between academia, industry and regulatory agencies is essential for developing safe and health-promoting products, as both prophylactic and therapeutic strategies.

\section{REFERENCES}

Asemi, Z., Zare, Z., Shakeri, H., Sabihi, S.-S., and Esmaillzadeh, A. (2013). Effect of multispecies probiotic supplements on metabolic profiles, Hs-CRP, and oxidative stress in patients with Type 2 diabetes. Ann. Nutr. Metab. 63, 1-9. doi: 10.1159/000349922

Atarashi, K., Tanoue, T., Oshima, K., Suda, W., Nagano, Y., Nishikawa, H., et al. (2013). Treg induction by a rationally selected mixture of Clostridia strains from the human microbiota. Nature 500, 232-236. doi: 10.1038/nature12331

Atarashi, K., Tanoue, T., Shima, T., Imaoka, A., Kuwahara, T., Momose, Y., et al. (2011). Induction of colonic regulatory $\mathrm{T}$ cells by indigenous Clostridium species. Science 331, 337-341. doi: 10.1126/science.1198469

Bakken, J. S. (2009). Fecal bacteriotherapy for recurrent Clostridium difficile infection. Anaerobe 15, 285-289. doi: 10.1016/j.anaerobe.2009.09.007

Bakken, J. S., Borody, T., Brandt, L. J., Brill, J. V., Demarco, D. C., Franzos, M. A., et al. (2011). Treating Clostridium difficile infection with fecal microbiota transplantation. Clin. Gastroenterol. Hepatol. 9, 1044-1049. doi: 10.1016/j.cgh. 2011.08.014

Baldi, A., and Arora, M. (2015). Regulatory categories of probiotics across the globe: a review representing existing and recommended categorization. Indian J. Med. Microbiol. 33(Suppl.), 2-10. doi: 10.4103/0255-0857.150868

Barbut, F., Richard, A., Hamadi, K., Chomette, V., Burghoffer, B., and Petit, J.-C. (2000). Epidemiology of recurrences or reinfections of Clostridium difficileassociated diarrhea. J. Clin. Microbiol. 38, 2386-2388.

Bartlett, J. G. (1990). Clostridium difficile: clinical considerations. Clin. Infect. Dis. 12(Suppl. 2), S243-S251. doi: 10.1093/clinids/12.supplement_2.s243

Becattini, S., Taur, Y., and Pamer, E. G. (2016). Antibiotic-induced changes in the intestinal microbiota and disease. Trends Mol. Med. 22, 458-478. doi: 10.1016/ j.molmed.2016.04.003

Besseling-van der Vaart, I., Heath, M. D., Guagnini, F., and Kramer, M. F. (2016). In vitro evidence for efficacy in food intolerance for the multispecies probiotic formulation Ecologic ${ }^{\circledR}$ Tolerance $\left(\right.$ Syngut ${ }^{\mathrm{TM}}$ ). Benef. Microbes 7, 111-118. doi: 10.3920/bm2015.0051

Brandt, L. J. (2012). Fecal transplantation for the treatment of Clostridium difficile infection. Gastroenterol. Hepatol. 8, 191-194.

Breyner, N. M., Michon, C., de Sousa, C. S., Vilas Boas, P. B., Chain, F., Azevedo, V. A., et al. (2017). Microbial anti-inflammatory molecule (MAM) from Faecalibacterium prausnitzii shows a protective effect on DNBS and DSSinduced colitis model in mice through inhibition of NF-кB pathway. Front. Microbiol. 8:114. doi: 10.3389/fmicb.2017.00114

Canani, R. B., Costanzo, M. D., Leone, L., Pedata, M., Meli, R., and Calignano, A. (2011). Potential beneficial effects of butyrate in intestinal and extraintestinal diseases. World J. Gastroenterol. 17, 1519-1528. doi: 10.3748/wjg.v17.i12. 1519

Cani, P. D., and Van Hul, M. (2015). Novel opportunities for next-generation probiotics targeting metabolic syndrome. Curr. Opin. Biotechnol. 32, 21-27. doi: 10.1016/j.copbio.2014.10.006

Chapman, C., Gibson, G., and Rowland, I. (2011). Health benefits of probiotics: are mixtures more effective than single strains? Eur. J. Nutr. 50, 1-17. doi: $10.1007 / \mathrm{s} 00394-010-0166-\mathrm{z}$

\section{AUTHOR CONTRIBUTIONS}

Conceived and designed the review: REH, EH-S, and TVW. Funding acquisition: TVW. Wrote the paper: REH. Reviewed the manuscript: REH, EH-S, and TVW.

\section{ACKNOWLEDGMENT}

The research leading to these results has received funding from the People Program (Marie Curie Actions) of the European Union's Seventh Framework Program FP7/2007-2013/under REA grant agreement $n^{\circ} 606713$.

Chen, J., Wang, R., Li, X.-F., and Wang, R.-L. (2011). Bifidobacterium adolescentis supplementation ameliorates visceral fat accumulation and insulin sensitivity in an experimental model of the metabolic syndrome. Br. J. Nutr. 107, 1429-1434. doi: 10.1017/s0007114511004491

Clarke, S. F., Murphy, E. F., O’Sullivan, O., Lucey, A. J., Humphreys, M., Hogan, A., et al. (2014). Exercise and associated dietary extremes impact on gut microbial diversity. Gut 63, 1913-1920. doi: 10.1136/gutjnl-2013-306541

Damin, M. R., Minowa, E., Alcântara, M. R., and Oliveira, M. N. (2008). Effect of cold storage on culture viability and some rheological properties of fermented milk prepared with yogurt and probiotic bacteria. J. Texture Stud. 39, 40-55. doi: 10.1111/j.1745-4603.2007.00129.x

Dao, M. C., Everard, A., Aron-Wisnewsky, J., Sokolovska, N., Prifti, E., Verger, E. O., et al. (2016). Akkermansia muciniphila and improved metabolic health during a dietary intervention in obesity: relationship with gut microbiome richness and ecology. Gut 65, 426-436. doi: 10.1136/gutjnl-2014308778

De Palma, G., Collins, S. M., and Bercik, P. (2014). The microbiota-gut-brain axis in functional gastrointestinal disorders. Gut Microbes 5, 419-429. doi: 10.4161/ gmic. 29417

De Palma, G., Lynch, M. D. J., Lu, J., Dang, V. T., Deng, Y., Jury, J., et al. (2017). Transplantation of fecal microbiota from patients with irritable bowel syndrome alters gut function and behavior in recipient mice. Sci. Transl. Med. 9:eaaf6397. doi: 10.1126/scitranslmed.aaf6397

den Besten, G., van Eunen, K., Groen, A. K., Venema, K., Reijngoud, D.-J., and Bakker, B. M. (2013). The role of short-chain fatty acids in the interplay between diet, gut microbiota, and host energy metabolism. J. Lipid Res. 54, 2325-2340. doi: 10.1194/jlr.r036012

Dethlefsen, L., and Relman, D. A. (2010). Incomplete recovery and individualized responses of the human distal gut microbiota to repeated antibiotic perturbation. Proc. Natl. Acad. Sci. U.S.A. 108(Suppl. 1), 4554-4561. doi: 10.1073/pnas.1000087107

Di Cerbo, A., Palmieri, B., Aponte, M., Morales-Medina, J. C., and Iannitti, T. (2016). Mechanisms and therapeutic effectiveness of lactobacilli. J. Clin. Pathol. 69, 187-203. doi: 10.1136/jclinpath-2015-202976

Distrutti, E., Monaldi, L., Ricci, P., and Fiorucci, S. (2016). Gut microbiota role in irritable bowel syndrome: new therapeutic strategies. World J. Gastroenterol. 22, 2219-2241. doi: 10.3748/wjg.v22.i7.2219

EFSA Panel on Biological Hazards (BIOHAZ) (2013). Scientific opinion on the maintenance of the list of QPS biological agents intentionally added to food and feed (2013 update). EFSA J. 11:3449. doi: 10.2903/j.efsa.2013.3449

Engels, C., Ruscheweyh, H.-J., Beerenwinkel, N., Lacroix, C., and Schwab, C. (2016). The common gut microbe Eubacterium hallii also contributes to intestinal propionate formation. Front. Microbiol. 7:713. doi: 10.3389/fmicb. 2016.00713

Evans, C. T., and Johnson, S. (2015). Prevention of Clostridium difficile infection with probiotics. Clin. Infect. Dis. 60(Suppl. 2), S122-S128. doi: 10.1093/cid/ civ138

Everard, A., Lazarevic, V., Gaïa, N., Johansson, M., Ståhlman, M., Backhed, F., et al. (2014). Microbiome of prebiotic-treated mice reveals novel targets involved in host response during obesity. ISME J. 8, 2116-2130. doi: 10.1038/ismej.2014.45 
Fernández-Murga, M. L., and Sanz, Y. (2016). Safety assessment of Bacteroides uniformis CECT 7771 isolated from stools of healthy breast-fed infants. PLOS ONE 11:e0145503. doi: 10.1371/journal.pone.0145503

Figueroa, I., Johnson, S., Sambol, S. P., Goldstein, E. J. C., Citron, D. M., and Gerding, D. N. (2012). Relapse versus reinfection: recurrent Clostridium difficile infection following treatment with fidaxomicin or vancomycin. Clin. Infect. Dis. 55(Suppl. 2), S104-S109. doi: 10.1093/cid/cis357

Francino, M. P. (2016). Antibiotics and the human gut microbiome: dysbioses and accumulation of resistances. Front. Microbiol. 6:1543. doi: 10.3389/fmicb.2015. 01543

Gauffin Cano, P., Santacruz, A., Moya, Á., and Sanz, Y. (2012). Bacteroides uniformis CECT 7771 ameliorates metabolic and immunological dysfunction in mice with high-fat-diet induced obesity. PLOS ONE 7:e41079. doi: 10.1371/ journal.pone.0041079

Gibson, G. R., Hutkins, R., Sanders, M. E., Prescott, S. L., Reimer, R. A., Salminen, S. J., et al. (2017). Expert consensus document: the international scientific association for probiotics and prebiotics (ISAPP) consensus statement on the definition and scope of prebiotics. Nat. Rev. Gastroenterol. Hepatol. 14, 491-502. doi: 10.1038/nrgastro.2017.75

Goldenberg, J. Z., Ma, S. S. Y., Saxton, J. D., Martzen, M. R., Vandvik, P. O., Thorlund, K., et al. (2013). Probiotics for the prevention of Clostridium difficile-associated diarrhea in adults and children. Cochrane Database Syst. Rev. doi: 10.1002/14651858.CD006095.pub3

Govender, M., Choonara, Y. E., Kumar, P., du Toit, L. C., van Vuuren, S., and Pillay, V. (2013). A review of the advancements in probiotic delivery: conventional vs. non-conventional formulations for intestinal flora supplementation. AAPS PharmsciTech 15, 29-43. doi: 10.1208/s12249013-0027-1

Graf, D., Di Cagno, R., Fåk, F., Flint, H. J., Nyman, M., Saarela, M., et al. (2015). Contribution Of diet to the composition of the human gut microbiota. Microb. Ecol. Health Dis. 26:26164. doi: 10.3402/mehd.v26.26164

Guarner, F., and Malagelada, J.-R. (2003). Gut flora in health and disease. Lancet 361, 512-519. doi: 10.1016/s0140-6736(03)12489-0

Gueimonde, M., and Sánchez, B. (2012). Enhancing probiotic stability in industrial processes. Microb. Ecol. Health Dis. 23:18562. doi: 10.3402/mehd.v23i0.18562

Hand, T. W. (2016). The role of the microbiota in shaping infectious immunity. Trends Immunol. 37, 647-658. doi: 10.1016/j.it.2016.08.007

Hartstra, A. V., Bouter, K. E. C., Bäckhed, F., and Nieuwdorp, M. (2015). Insights into the role of the microbiome in obesity and type 2 diabetes. Diabetes Care 38 , 159-165. doi: 10.2337/dc14-0769

Heidebach, T., Först, P., and Kulozik, U. (2012). Microencapsulation of probiotic cells for food applications. Crit. Rev. Food Sci. Nutr. 52, 291-311. doi: 10.1080/ 10408398.2010.499801

Hill, C., Guarner, F., Reid, G., Gibson, G. R., Merenstein, D. J., Pot, B., et al. (2014). Expert consensus document: the international scientific association for probiotics and prebiotics consensus statement on the scope and appropriate use of the term probiotic. Nat. Rev. Gastroenterol. Hepatol. 11, 506-514. doi: $10.1038 /$ nrgastro.2014.66

Jafarnejad, S., Saremi, S., Jafarnejad, F., and Arab, A. (2016). Effects of a multispecies probiotic mixture on glycemic control and inflammatory status in women with gestational diabetes: a randomized controlled clinical trial. J. Nutr. Metab. 2016:5190846. doi: 10.1155/2016/5190846

Jiang, Z.-D., Hoang, L. N., Lasco, T. M., Garey, K. W., and DuPont, H. L. (2013). Physician attitudes toward the use of fecal transplantation for recurrent Clostridium difficile infection in a metropolitan area. Clin. Infect. Dis. 56, 1059-1060. doi: 10.1093/cid/cis1025

Johnson, S., Adelmann, A., Clabots, C. R., Peterson, L. R., and Gerding, D. N. (1989). Recurrences of Clostridium difficile diarrhea not caused by the original infecting organism. J. Infect. Dis. 159, 340-343. doi: 10.1093/infdis/159.2.340

Kasińska, M. A., and Drzewoski, J. (2015). Effectiveness of probiotics in Type 2 diabetes: a meta-analysis. Pol. Arch. Intern. Med. 125, 803-813. doi: 10.20452/ pamw.3156

Kelly, C. P. (2009). A 76-year-old man with recurrent Clostridium difficile-associated diarrhea. JAMA 301, 954-962. doi: 10.1001/jama.200 9.171

Kelly, C. P. (2013). Fecal microbiota transplantation-an old therapy comes of age. N. Engl. J. Med. 368, 474-475. doi: 10.1056/nejme1214816
Khanna, S., Pardi, D. S., Kelly, C. R., Kraft, C. S., Dhere, T., Henn, M. R., et al. (2016). A novel microbiome therapeutic increases gut microbial diversity and prevents recurrent Clostridium difficile infection. J. Infect. Dis. 214, 173-181. doi: 10.1093/infdis/jiv766

Kim, S.-W., Park, K.-Y., Kim, B., Kim, E., and Hyun, C.-K. (2013). Lactobacillus rhamnosus GG improves insulin sensitivity and reduces adiposity in highfat diet-fed mice through enhancement of adiponectin production. Biochem. Biophys. Res. Commun. 431, 258-263. doi: 10.1016/j.bbrc.2012.12.121

Klaenhammer, T. R., Kleerebezem, M., Kopp, M. V., and Rescigno, M. (2012). The impact of probiotics and prebiotics on the immune system. Nat. Rev. Immunol. 12, 728-734. doi: 10.1038/nri3312

Kobyliak, N., Conte, C., Cammarota, G., Haley, A. P., Styriak, I., Gaspar, L., et al. (2016). Probiotics in prevention and treatment of obesity: a critical view. Nutr. Metab. 13, 14. doi: 10.1186/s12986-016-0067-0

Koning, C. J., Jonkers, D. M., Stobberingh, E. E., Mulder, L., Rombouts, F. M., and Stockbrügger, R. W. (2008). The effect of a multispecies probiotic on the intestinal microbiota and bowel movements in healthy volunteers taking the antibiotic amoxycillin. Am. J. Gastroenterol. 103, 178-189. doi: 10.1111/j.15720241.2007.01547.x

Kristensen, N. B., Bryrup, T., Allin, K. H., Nielsen, T., Hansen, T. H., and Pedersen, O. (2016). Alterations in fecal microbiota composition by probiotic supplementation in healthy adults: a systematic review of randomized controlled trials. Genome Med. 8, 52. doi: 10.1186/s13073-016-0300-5

Kwon, H.-K., Lee, C.-G., So, J.-S., Chae, C.-S., Hwang, J.-S., Sahoo, A., et al. (2010). Generation of regulatory dendritic cells and $\mathrm{CD} 4^{+} \mathrm{Foxp}^{+} \mathrm{T}$ cells by probiotics administration suppresses immune disorders. Proc. Natl. Acad. Sci. U.S.A. 107, 2159-2164. doi: 10.1073/pnas.0904055107

Langdon, A., Crook, N., and Dantas, G. (2016). The effects of antibiotics on the microbiome throughout development and alternative approaches for therapeutic modulation. Genome Med. 8:39. doi: 10.1186/s13073-016-0294-z

Leszczyszyn, J. J., Radomski, M., and Leszczyszyn, A. M. (2016). Intestinal microbiota transplant - current state of knowledge. Reumatologia 54, 24-28. doi: 10.5114/reum.2016.58758

Li, J., Lin, S., Vanhoutte, P. M., Woo, C. W., and Xu, A. (2016). Akkermansia muciniphila protects against atherosclerosis by preventing metabolic endotoxemia-induced inflammation in apoe-/-mice clinical. Circulation 133, 2434-2446. doi: 10.1161/circulationaha.115.019645

Lowes, R. (2016). FDA Proposes Tighter Rules For Fecal Transplants. New York, NY: Medscape.

Macpherson, A. J., de Agüero, M. G., and Ganal-Vonarburg, S. C. (2017). How nutrition and the maternal microbiota shape the neonatal immune system. Nat. Rev. Immunol. 17, 508-517. doi: 10.1038/nri.2017.58

Marotz, C. A., and Zarrinpar, A. (2016). Treating obesity and metabolic syndrome with fecal microbiota transplantation. Yale J. Biol. Med. 89, 383-388.

Marsh, J. W., Arora, R., Schlackman, J. L., Shutt, K. A., Curry, S. R., and Harrison, L. H. (2012). Association of relapse of Clostridium difficile disease with BI/NAP1/027. J. Clin. Microbiol. 50, 4078-4082. doi: 10.1128/jcm. 02291-12

Martín, R., Chain, F., Miquel, S., Lu, J., Gratadoux, J.-J., Sokol, H., et al. (2014). The commensal bacterium Faecalibacterium prausnitzii is protective in DNBSinduced chronic moderate and severe colitis models. Inflamm. Bowel Dis. 20, 417-430. doi: 10.1097/01.mib.0000440815.76627.64

Martín, R., Miquel, S., Benevides, L., Bridonneau, C., Robert, V., Hudault, S., et al. (2017). Functional characterization of novel Faecalibacterium prausnitzii strains isolated from healthy volunteers: a step forward in the use of F. prausnitzii as a next-generation probiotic. Front. Microbiol. 8:1226. doi: $10.3389 /$ fmicb.2017.01226

Miettinen, M., Vuopio-Varkila, J., and Varkila, K. (1996). Production of human tumor necrosis factor alpha, interleukin-6, and interleukin-10 is induced by lactic acid bacteria. Infect. Immun. 64, 5403-5405.

Miquel, S., Leclerc, M., Martin, R., Chain, F., Lenoir, M., Raguideau, S., et al. (2015). Identification of metabolic signatures linked to anti-inflammatory effects of Faecalibacterium prausnitzii. mBio 6:e00300-15. doi: 10.1128/mbio.00300-15

Moayyedi, P., Surette, M. G., Kim, P. T., Libertucci, J., Wolfe, M., Onischi, C., et al. (2015). Fecal microbiota transplantation induces remission in patients with active ulcerative colitis in a randomized controlled trial. Gastroenterology 149, 102.e6-109.e6. doi: 10.1053/j.gastro.2015.04.001 
Nazemian, V., Shadnoush, M., Manaheji, H., and Zaringhalam, J. (2016). Probiotics and inflammatory pain: a literature review study. Middle East J. Rehabil. Health Stud. 3:e36087. doi: 10.17795/mejrh-36087

Neef, A., and Sanz, Y. (2013). Future for probiotic science in functional food and dietary supplement development. Curr. Opin. Clin. Nutr. Metab. Care 16, 679-687. doi: 10.1097/mco.0b013e328365c258

Ng, S. C., Hart, A. L., Kamm, M. A., Stagg, A. J., and Knight, S. C. (2009). Mechanisms of action of probiotics: recent advances. Inflamm. Bowel Dis. 15, 300-310. doi: 10.1002/ibd.20602

Orenstein, R., Dubberke, E., Hardi, R., Ray, A., Mullane, K., Pardi, D. S., et al. (2015). Safety and durability of RBX2660 (microbiota suspension) for recurrent Clostridium difficile infection: results of the PUNCH CD study. Clin. Infect. Dis. 62, 596-602. doi: 10.1093/cid/civ938

Patel, R., and DuPont, H. L. (2015). New approaches for bacteriotherapy: prebiotics, new-generation probiotics, and synbiotics. Clin. Infect. Dis. 60(Suppl. 2), S108-S121. doi: 10.1093/cid/civ177

Patrascu, O., Béguet-Crespel, F., Marinelli, L., Le Chatelier, E., Abraham, A. L., Leclerc, M., et al. (2017). A fibrolytic potential in the human ileum mucosal microbiota revealed by functional metagenomic. Sci. Rep. 7:40248. doi: 10.1038/ srep 40248

Pattani, R., Palda, V. A., Hwang, S. W., and Shah, P. S. (2013). Probiotics for the prevention of antibiotic-associated diarrhea and Clostridium difficile infection among hospitalized patients: systematic review and meta-analysis. Open Med. 7, 56-67.

Paulo Sousa e Silva, J., and Freitas, A. C. (2014). Probiotic Bacteria, 1st Edn. Singapore: Pan Stanford Publishing.

Pérez-Cobas, A., Moya, A., Gosalbes, M., and Latorre, A. (2015). Colonization resistance of the gut microbiota against Clostridium difficile. Antibiotics 4, 337-357. doi: 10.3390/antibiotics4030337

Petrof, E. O., Gloor, G. B., Vanner, S. J., Weese, S. J., Carter, D., Daigneault, M. C., et al. (2013). Stool substitute transplant therapy for the eradication of Clostridium difficile infection: 'RePOOPulating' the gut. Microbiome 1:3. doi: 10.1186/2049-2618-1-3

Pharmabiotic Research Institute (2017). PRI: Microbiotic Medicinal Products. Available at: http://www.pharmabiotic.org/

Pigneur, B., and Sokol, H. (2016). Fecal microbiota transplantation in inflammatory bowel disease: the quest for the holy grail. Mucosal Immunol. 9, 1360-1365. doi: $10.1038 / \mathrm{mi} .2016 .67$

Pinto-Sanchez, M. I., Hall, G. B., Ghajar, K., Nardelli, A., Bolino, C., Lau, J. T., et al. (2017). Probiotic Bifidobacterium longum NCC3001 reduces depression scores and alters brain activity: a pilot study in patients with irritable bowel syndrome. Gastroenterology 153, 448.e8-459.e8. doi: 10.1053/j.gastro.2017. 05.003

Plaza-Diaz, J. (2014). Modulation of immunity and inflammatory gene expression in the gut, in inflammatory diseases of the gut and in the liver by probiotics. World J. Gastroenterol. 20:15632. doi: 10.3748/wjg.v20.i42.15632

Plaza-Diaz, J., Gomez-Llorente, C., Abadia-Molina, F., Saez-Lara, M. J., CampañaMartin, L., Muñoz-Quezada, S., et al. (2014). Effects of Lactobacillus paracasei CNCM I-4034, Bifidobacterium breve CNCM I-4035 and Lactobacillus rhamnosus CNCM I-4036 on hepatic steatosis in zucker rats. PLOS ONE 9:e98401. doi: 10.1371/journal.pone.0098401

Quévrain, E., Maubert, M. A., Michon, C., Chain, F., Marquant, R., Tailhades, J., et al. (2016). Identification of an anti-inflammatory protein from Faecalibacterium prausnitzii, a commensal bacterium deficient in Crohn's disease. Gut 65, 415-425. doi: 10.1136/gutjnl-2014-307649

Ramesh, M., Khanna, S., Messer, J., and Adams, M. (2016). Prevention of Recurrent C. difficile Infection with RBX2660 | PUNCH CD 2 Results. Roseville, MN: Rebiotix.

Rao, K., and Safdar, N. (2015). Fecal microbiota transplantation for the treatment of Clostridium difficile infection. J. Hosp. Med. 11, 56-61. doi: 10.1002/jhm. 2449

Rao, K., and Young, V. B. (2017). Probiotics for prevention of Clostridium difficile infection in hospitalized patients: is the jury still out? Gastroenterology 152, 1817-1819. doi: 10.1053/j.gastro.2017.04.027

Ratner, M. (2015). Microbial cocktails join fecal transplants in IBD treatment trials. Nat. Biotechnol. 33, 787-788. doi: 10.1038/nbt0815-787

Reardon, S. (2014). Microbiome therapy gains market traction. Nature 509, 269-270. doi: $10.1038 / 509269$ a
Rebiotix Media (2015). Rebiotix Receives Breakthrough Therapy Designation for RBX2660 - A Microbiota Restoration Therapy (MRT) for the Treatment of Recurrent Clostridium difficile Infection. Available at: http://www.rebiotix.com/wp-content/uploads/rebiotix_receives_ breakthrough_therapy_designation_recurrent_clostridium_difficile.pdf

Reichold, A., Brenner, S. A., Spruss, A., Förster-Fromme, K., Bergheim, I., and Bischoff, S. C. (2014). Bifidobacterium adolescentis protects from the development of nonalcoholic steatohepatitis in a mouse model. J. Nutr. Biochem. 25, 118-125. doi: 10.1016/j.jnutbio.2013.09.011

Reijnders, D., Goossens, G. H., Hermes, G. D., Neis, E. P., van der Beek, C. M., Most, J., et al. (2016). Effects of gut microbiota manipulation by antibiotics on host metabolism in obese humans: a randomized double-blind placebocontrolled trial. Cell Metab. 24, 63-74. doi: 10.1016/j.cmet.2016.07.008

Ricci, A., Allende, A., Bolton, D., Chemaly, M., Davies, R., Girones, R., et al. (2017). Update of the list of QPS-recommended biological agents intentionally added to food or feed as notified to EFSA 5: suitability of taxonomic units notified to EFSA until September 2016. EFSA J. 15:4663. doi: 10.2903/j.efsa.2017.4663

Rivière, A., Selak, M., Lantin, D., Leroy, F., and De Vuyst, L. (2016). Bifidobacteria and butyrate-producing colon bacteria: importance and strategies for their stimulation in the human gut. Front. Microbiol. 7:979. doi: 10.3389/fmicb.2016. 00979

Round, J. L., Lee, S. M., Li, J., Tran, G., Jabri, B., Chatila, T. A., et al. (2011). The tolllike receptor 2 pathway establishes colonization by a commensal of the human microbiota. Science 332, 974-977. doi: 10.1126/science.1206095

Round, J. L., and Mazmanian, S. K. (2009). The gut microbiota shapes intestinal immune responses during health and disease. Nat. Rev. Immunol. 9, 600-600. doi: $10.1038 /$ nri2614

Sanders, M. E. (2008). Probiotics: definition, sources, selection, and uses. Clin. Infect. Dis. 46, S58-S61. doi: 10.1086/523341

Sang, L.-X. (2010). Remission induction and maintenance effect of probiotics on ulcerative colitis: a meta-analysis. World J. Gastroenterol. 16:1908. doi: 10.3748/ wjg.v16.i15.1908

Saulnier, D. M., Ringel, Y., Heyman, M. B., Foster, J. A., Bercik, P., Shulman, R. J., et al. (2013). The intestinal microbiome, probiotics and prebiotics in neurogastroenterology. Gut Microbes 4, 17-27. doi: 10.4161/gmic.22973

Savcheniuk, O., Kobyliak, N., Kondro, M., Virchenko, O., Falalyeyeva, T., and Beregova, T. (2014). Short-term periodic consumption of multiprobiotic from childhood improves insulin sensitivity, prevents development of nonalcoholic fatty liver disease and adiposity in adult rats with glutamate-induced obesity. BMC Complement. Altern. Med. 14:247. doi: 10.1186/1472-688214-247

Sbahi, H., and Di Palma, J. A. (2016). Faecal microbiota transplantation: applications and limitations in treating gastrointestinal disorders. BMJ Open Gastroenterol. 3:e000087. doi: 10.1136/bmjgast-2016-000087

Schneeberger, M., Everard, A., Gómez-Valadés, A. G., Matamoros, S., Ramírez, S., Delzenne, N. M., et al. (2015). Akkermansia muciniphila inversely correlates with the onset of inflammation, altered adipose tissue metabolism and metabolic disorders during obesity in mice. Sci. Rep. 5:16643. doi: 10.1038/ srep 16643

Scott, K. P., Antoine, J. M., Midtvedt, T., and van Hemert, S. (2015). Manipulating the gut microbiota to maintain health and treat disease. Microb. Ecol. Health Dis. 26:25877. doi: 10.3402/mehd.v26.25877

Seres Therapeutics (2015). Seres Therapeutics, Inc. Announces FDA Orphan Drug Designation for SER-109 for the Prevention of Recurrent Clostridium difficile Infection in Adults. Available at: http://ir.serestherapeutics.com/phoenix.zhtml? $\mathrm{c}=254006 \& \mathrm{p}=$ irol-newsArticle\&ID $=2081167$

Seres Therapeutics (2017). Inflammatory Bowel Disease. Serestherapeutics.Com. Available at: http://www.serestherapeutics.com/pipeline/products/ inflammatory-bowel-disease

Shah, N. P., Ding, W. K., Fallourd, M. J., and Leyer, G. (2010). Improving the stability of probiotic bacteria in model fruit juices using vitamins and antioxidants. J. Food Sci. 75, M278-M282. doi: 10.1111/j.1750-3841.2010. 01628.x

Shi, Y., Dong, Y., Huang, W., Zhu, D., Mao, H., and Su, P. (2016). Fecal microbiota transplantation for ulcerative colitis: a systematic review and meta-analysis. PLOS ONE 11:e0157259. doi: 10.1371/journal.pone.0157259

Sokol, H., Galperine, T., Kapel, N., Bourlioux, P., Seksik, P., Barbut, F., et al. (2016). Faecal microbiota transplantation in recurrent Clostridium difficile infection: 
recommendations from the French group of faecal microbiota transplantation. Dig. Liver Dis. 48, 242-247. doi: 10.1016/j.dld.2015.08.017

Sokol, H., Pigneur, B., Watterlot, L., Lakhdari, O., Bermudez-Humaran, L. G., Gratadoux, J.-J., et al. (2008). Faecalibacterium prausnitzii is an antiinflammatory commensal bacterium identified by gut microbiota analysis of Crohn disease patients. Proc. Natl. Acad. Sci. U.S.A. 105, 16731-16736. doi: $10.1073 /$ pnas.0804812105

Steenbergen, L., Sellaro, R., van Hemert, S., Bosch, J. A., and Colzato, L. S. (2015). A randomized controlled trial to test the effect of multispecies probiotics on cognitive reactivity to sad mood. Brain Behav. Immun. 48, 258-264. doi: 10.1016/j.bbi.2015.04.003

Surawicz, C. M., Brandt, L. J., Binion, D. G., Ananthakrishnan, A. N., Curry, S. R., Gilligan, P. H., et al. (2013). Guidelines for diagnosis, treatment, and prevention of Clostridium difficile infections. Am. J. Gastroenterol. 108, 478-498. doi: 10.1038/ajg.2013.4

Timmerman, H. M., Koning, C. J. M., Mulder, L., Rombouts, F. M., and Beynen, A. C. (2004). Monostrain, multistrain and multispecies probiotics-a comparison of functionality and efficacy. Int. J. Food Microbiol. 96, 219-233. doi: 10.1016/j.ijfoodmicro.2004.05.012

Timmerman, H. M., Niers, L. E. M., Ridwan, B. U., Koning, C. J. M., Mulder, L., Akkermans, L. M. A., et al. (2007). Design of a multispecies probiotic mixture to prevent infectious complications in critically ill patients. Clin. Nutr. 26, 450-459. doi: 10.1016/j.clnu.2007.04.008

Troy, E. B., and Kasper, D. L. (2010). Beneficial effects of Bacteroides fragilis polysaccharides on the immune system. Front. Biosci. 15, 25-34. doi: 10.2741/ 3603

Udayappan, S., Manneras-Holm, L., Chaplin-Scott, A., Belzer, C., Herrema, H., Dallinga-Thie, G.-M., et al. (2016). Oral treatment with Eubacterium hallii improves insulin sensitivity in $\mathrm{db} / \mathrm{db}$ mice. NPJ Biofilms Microbiomes 2:16009. doi: 10.1038/npjbiofilms.2016.9

van der Lelie, D., Taghavi, S., Henry, C., and Gilbert, J. A. (2017). The microbiome as a source of new enterprises and job creation: considering clinical faecal and synthetic microbiome transplants and therapeutic regulation. Microb. Biotechnol. 10, 4-5. doi: 10.1111/1751-7915.12597

van Minnen, L. P., Timmerman, H. M., Lutgendorff, F., Verheem, A., Harmsen, W., Konstantinov, S. R., et al. (2007). Modification of intestinal flora with multispecies probiotics reduces bacterial translocation and improves clinical course in a rat model of acute pancreatitis. Surgery 141, 470-480. doi: 10.1016/ j.surg.2006.10.007

van Nood, E., Speelman, P., Nieuwdorp, M., and Keller, J. (2014). Fecal Microbiota Transplantation. New York, NY: Medscape.

Venturi, A., Gionchetti, P., Rizzello, F., Johansson, R., Zucconi, E., Brigidi, P., et al. (1999). Impact on the composition of the faecal flora by a new probiotic preparation: preliminary data on maintenance treatment of patients with ulcerative colitis. Aliment. Pharmacol. Ther. 13, 1103-1108. doi: 10.1046/j.13652036.1999.00560.x

Vermeire, S., Joossens, M., Verbeke, K., Wang, J., Machiels, K., Sabino, J., et al. (2015). Donor species richness determines faecal microbiota transplantation success in inflammatory bowel disease. J. Crohns Colitis 10, 387-394. doi: $10.1093 /$ ecco-jcc/jjv203

Wallace, C. J. K., and Milev, R. (2017). The effects of probiotics on depressive symptoms in humans: a systematic review. Ann. Gen. Psychiatry 16, 14. doi: 10.1186/s12991-017-0141-7

Wang, J., Tang, H., Zhang, C., Zhao, Y., Derrien, M., Rocher, E., et al. (2014). Modulation of gut microbiota during probiotic-mediated attenuation of metabolic syndrome in high fat diet-fed mice. ISME J. 9, 1-15. doi: 10.1038/ ismej.2014.99

Wexler, H. M. (2007). Bacteroides: the good, the bad, and the nitty-gritty. Clin. Microbiol. Rev. 20, 593-621. doi: 10.1128/cmr.00008-07

WHO/FAO (2006). Probiotics in Food Health and Nutritional Properties and Guidelines for Evaluation. Rome: Food and Agriculture Organization of the United Nations.

Yan, F., and Polk, D. B. (2011). Probiotics and immune health. Curr. Opin. Gastroenterol. 27, 496-501. doi: 10.1097/mog.0b013e32834baa4d

Yang, J.-Y., Lee, Y.-S., Kim, Y., Lee, S.-H., Ryu, S., Fukuda, S., et al. (2016). Gut Commensal Bacteroides acidifaciens prevents obesity and improves insulin sensitivity in mice. Mucosal Immunol. 10, 104-116. doi: 10.1038/mi.2016.42

Yin, Y.-N. (2010). Effects of four Bifidobacteria on obesity in high-fat diet induced rats. World J. Gastroenterol. 16:3394. doi: 10.3748/wjg.v16.i27. 3394

Yoon, J. S., Sohn, W., Lee, O. Y., Lee, S. P., Lee, K. N., Jun, D. W., et al. (2013). Effect of multispecies probiotics on irritable bowel syndrome: a randomized, double-blind, placebo-controlled trial. J. Gastroenterol. Hepatol. 29, 52-59. doi: 10.1111 /jgh. 12322

Youngster, I., Russell, G., Pindar, C., Sauk, J., and Hohmann, E. (2014). 17980ral, frozen fecal microbiota capsules for relapsing Clostridium difficile infection. Open Forum Infect. Dis. 1(Suppl. 1), S62-S62. doi: 10.1093/ofid/ ofu051.170

Zhou, D. (2016). Impact of sanitary living environment on gut microbiota. Precis. Med. 2:e1161. doi: 10.14800/pm.1161

Zipursky, J. S., Sidorsky, T. I., Freedman, C. A., Sidorsky, M. N., and Kirkland, K. B. (2014). Patient attitudes toward the use of fecal microbiota transplantation in the treatment of recurrent Clostridium difficile infection. Clin. Infect. Dis. 55, 1652-1658. doi: 10.1093/cid/cis809

Conflict of Interest Statement: The authors declare that the research was conducted in the absence of any commercial or financial relationships that could be construed as a potential conflict of interest.

Copyright (c) 2017 El Hage, Hernandez-Sanabria and Van de Wiele. This is an open-access article distributed under the terms of the Creative Commons Attribution License (CC BY). The use, distribution or reproduction in other forums is permitted, provided the original author(s) or licensor are credited and that the original publication in this journal is cited, in accordance with accepted academic practice. No use, distribution or reproduction is permitted which does not comply with these terms. 